Polymer Journal, Vol. 39, No. 5, pp. 458-463 (2007)

(C) 2007 The Society of Polymer Science, Japan

\title{
Direction of Temperature Gradient and Retention Mechanism in Normal-Phase Temperature Gradient Interaction Chromatography for Poly(methyl methacrylate) Fractionation
}

\author{
Hiroshi Matsumoto,$^{\dagger}$ Tadatomo Kawai, and Shinya TeramaChi \\ Department of Materials Science and Technology, Faculty of Engineering, Kogakuin University, \\ 2665-1 Nakano-cho, Hachioji 192-0015, Japan
}

(Received December 1, 2006; Accepted February 20, 2007; Published March 30, 2007)

\begin{abstract}
Temperature gradient interaction chromatography (TGIC) is a powerful technique for molecular characterization of polymers, in which the interaction strength is controlled by varying the column temperature. In the present paper, the direction of temperature gradient for normal-phase mode TGIC in poly(methyl methacrylate) (PMMA) fractionation was studied by using System I (column: cyano-modified silica // eluent: 1,4 -dioxane $/ n$-hexane) and System II (bare silica // 1,4-dioxane $/ \mathrm{CH}_{2} \mathrm{Cl}_{2}$ ), respectively. It was found that PMMA samples were separated by positive and negative temperature gradients using the respective systems. The results were also discussed theoretically. [doi:10.1295/polymj.PJ2006181]

KEY WORDS Temperature Gradient Interaction Chromatography / Normal-Phase / Direction of Temperature Gradient / Poly(methyl methacrylate) / Fractionation /
\end{abstract}

Temperature gradient interaction chromatography (TGIC) is a powerful technique for molecular characterization of polymers. ${ }^{1,2}$ TGIC separates polymer components in the order of molecular weight with a resolution superior to size exclusion chromatography (SEC). TGIC separation is driven mainly by adsorptive interaction between solutes and stationary phase and the strength of the interaction is controlled by varying the column temperature, to offset the molecular weight dependency of the adsorption strength. During the sample elution, the column temperature is varied continuously by positive (rising) or negative (lowering) temperature gradient. In many studies of TGIC, reversed-phase mode TGIC (RP-TGIC) has been applied to the separation of various polymers. In almost all cases of RP-TGIC, the positive temperature gradient was applied. The exception reported so far was a case where poly(ethylene oxide) (PEO) was fractionated using $\mathrm{C} 18$ bonded silica column and a mixture of acetonitrile and water by negative temperature gradient. ${ }^{2-4}$ On the other hand, the results in the normal-phase mode TGIC (NP-TGIC) reported are not so many. However both temperature gradients were reported in NP-TGIC. That is, polystyrene (PS), ${ }^{5,6}$ polyisoprene (PI) ${ }^{5} \mathrm{PEO}^{4}$ and poly(2-vinylpyridine) $(\mathrm{P} 2 \mathrm{VP})^{7}$ were separated by the positive temperature gradient, whereas PS samples were separated also by the negative temperature gradient using the respective columns of bare silica and $\mathrm{NH}_{2}$-modified silica packing materials and the eluent mixtures of chloroform and $n$-hexane $(n \mathrm{Hx})$ at the respective compositions in our previous work. ${ }^{6}$ It was reported that the positive temperature dependencies of the retention were obtained for $\mathrm{PS}^{8}$ and poly(styrene-co-methyl methacrylate $)[\mathrm{P}(\mathrm{S}-c o-\mathrm{MMA})]^{9}$ in common organic eluents and also poly(ethylene glycol) in aqueous eluents. ${ }^{10}$ If these systems were used for NP-TGIC, the negative temperature gradient should be effective. Then, it is very interesting whether not only low polar polymer PS but also high polar polymer poly(methyl methacrylate) (PMMA) can be separated by both of positive and negative temperature gradients in NPTGIC. All cases of TGIC separation of PMMA samples reported so far were carried out by RP-mode using positive temperature gradient, as far as we know. However, PMMA samples and block copolymer samples involving PMMA block were separated mainly by NP-mode in the liquid chromatography at the critical condition (LCCC), ${ }^{11,12}$ and the above mentioned case of $\mathrm{P}(\mathrm{S}-\mathrm{co}$-MMA) was also done by NP-mode of solvent gradient elution. Therefore the separation of PMMA samples using the NP-TGIC may be possible by choosing the appropriate eluent mixture.

In the present work, therefore, we studied the direction of temperature gradient for NP-TGIC in PMMA fractionation using common organic mobile phase, and found that PMMA samples were separated by both temperature gradients. The results were discussed also theoretically.

${ }^{\dagger}$ To whom correspondence should be addressed (Tel: +81-426-28-4619, Fax: +81-426-28-5647, E-mail: bd04003@ns.kogakuin.ac.jp). 


\section{EXPERIMENTAL}

The apparatus for TGIC was a typical HPLC system except for a modification to control precisely the temperature of a separation column. A long stainless tubing is installed between the pump (Agilent, 1100 series, G1312A) and the injector (Rheodyne, 7725 ) to pre-equilibrate the temperature of the eluent before it reaches the column. The separation column and the preheating tube were immersed in a temperature program bath (EYELA, PRO COOL BATH NCB 3200) to control the temperature.

Two types of separation columns were used, a cyano-modified silica column (Waters, $\mu$-bondasphere, particle size $5 \mu \mathrm{m}$, pore size $10 \mathrm{~nm}, 150 \times 3.9 \mathrm{~mm}$ I.D.) and a bare silica column (Chemco, Nucleosil, particle size $7 \mu \mathrm{m}$, pore size $10 \mathrm{~nm}, 150 \times 4.0 \mathrm{~mm}$ I.D.). The mobile phase were mixtures of 1,4-dioxane (Kanto Chemical, Premium grade) $/ n \mathrm{Hx}$ (Taisei Chemical, Premium grade) or 1,4-dioxane/dichloromethane $\left(\mathrm{CH}_{2} \mathrm{Cl}_{2}\right)$ (Wako Pure Chemical Industries, HPLC grade). 1,4-dioxane was purified by distillation after passing through molecular sieves $5 \mathrm{~A}$ to remove residual moisture. The flow rate of mobile phase was $0.3 \mathrm{~mL} / \mathrm{min}$.

Four standard PMMA samples were obtained from SHOWA DENKO K.K. (Japan), commercially. Because the elution behavior of PMMA is affected by the tacticity of the sample, ${ }^{13}$ the triad tacticities of PMMA samples were determined by the intensity measurements of the $\alpha$-methyl signals in the ${ }^{1} \mathrm{H}$ NMR (JEOL, EX270) spectra measured in chloroform- $d_{1}$ at room temperature and only the samples with near values of tacticity were used. The sample numbers, the weight-average molecular weights, the polydispersity indices $\left(M_{\mathrm{w}} / M_{\mathrm{n}}\right)$ and the tacticities were summarized in Table I. The sample solutions were filtered with a $0.50 \mu \mathrm{m}$ membrane filter (Advantec, DISMIC-3) and then injected through a 7725 Rheodyne injector with a $20 \mu \mathrm{L}$ sample loop. The chromatograms were recorded with an evaporative light scattering detector (ELSD) (Polymer laboratories, PL-ELS 1000) operated at nebulizer gas flow $1.3 \mathrm{~L} / \mathrm{min}$, nebulizer temperature $90^{\circ} \mathrm{C}$ and evaporator temperature $130^{\circ} \mathrm{C}$.

Table I. Poly(methyl methacrylate) standard samples ${ }^{\mathrm{a}}$

\begin{tabular}{lcccccc}
\hline \multicolumn{2}{c}{ Sample No. } & \multirow{2}{*}{$M_{\mathrm{w}}$} & $M_{\mathrm{w}} / M_{\mathrm{n}}$ & \multicolumn{4}{c}{ Tacticity $^{\mathrm{b}}$} \\
\cline { 5 - 7 } & & & & $m m$ & $m r$ & $r r$ \\
\hline PMMA & 1 & $2.23 \times 10^{4}$ & 1.02 & 1 & 16 & 83 \\
& 2 & $4.84 \times 10^{4}$ & 1.02 & 1 & 17 & 82 \\
& 3 & $2.15 \times 10^{5}$ & 1.02 & 2 & 14 & 84 \\
& 4 & $6.73 \times 10^{5}$ & 1.03 & 1 & 17 & 82 \\
\hline
\end{tabular}

${ }^{\mathrm{a}}$ From SHOWA DENKO K.K. ${ }^{\mathrm{b}}$ Determined by ${ }^{1} \mathrm{H}$ NMR

\section{RESULTS AND DISCUSSION}

TGIC separations of four PMMA standards were carried out by using a cyano-modified silica column and a mixture of 1,4-dioxane and $n \mathrm{Hx}$ (System I: $\mathrm{CN} / / 1,4$-dioxane $/ n \mathrm{Hx}$ ), and a bare silica column and a mixture of 1,4-dioxane and $\mathrm{CH}_{2} \mathrm{Cl}_{2}$ (System II: Silica // 1,4-dioxane/ $\mathrm{CH}_{2} \mathrm{Cl}_{2}$ ), respectively. In the Figure 1, ELSD chromatograms for a mixture of four PMMA standard samples obtained by System I using the various compositions (1,4-dioxane content was labeled in the figure) at constant temperature, $30^{\circ} \mathrm{C}$. The mixture containing 66 vol. \% of 1,4-dioxane appeared to be around the critical composition. In Figure 2, the chromatograms obtained using the mixture (1,4-dioxane $/ n \mathrm{Hx}=66 / 34, \mathrm{v} / \mathrm{v})$ as eluent at different temperatures are displayed. In these measurements, sample solutions were prepared with pure 1,4-dioxane at the concentration of about $0.25 \mathrm{mg} /$ $\mathrm{mL}$ totally. (In TGIC measurements, the samples were dissolved in the mixed solvents at the critical compositions for the respective columns.) At higher temperature (above $30^{\circ} \mathrm{C}$ ), PMMA samples were eluted out at earlier elution time $(2-5 \mathrm{~min})$, on the other hand, at the lower temperature (below $30^{\circ} \mathrm{C}$ ), the samples were retained strongly $(5 \mathrm{~min} \sim)$. Therefore these results suggested that the temperature dependence of retention is negative $\left(\partial V_{\mathrm{R}} / \partial T\right)_{P}<0$ and then a positive temperature gradient is effective for the molecular

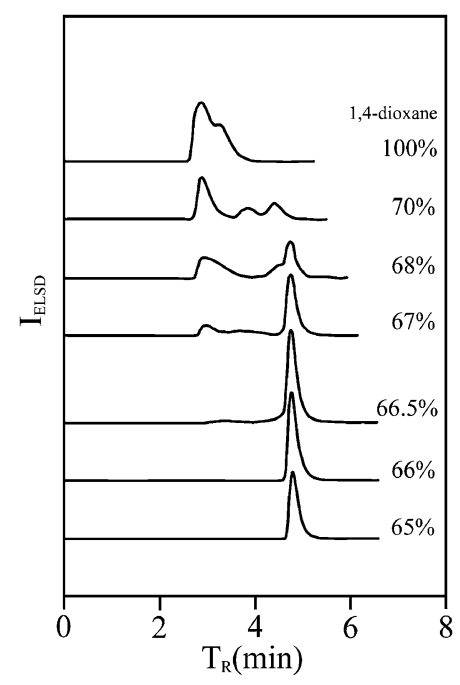

Figure 1. Solvent composition effect on the chromatograms of 4 PMMA standard samples by System I. The isocratic elution of 1,4-dioxane $/ n$-hexane at different compositions as labeled in the figure were tested at a constant temperature, $30^{\circ} \mathrm{C}$, to find the critical condition. A cyano-modified silica column (Waters, $\mu$-bondasphere, particle size $5 \mu \mathrm{m}$, pore size $10 \mathrm{~nm}, 150 \times 3.9 \mathrm{~mm}$ I.D.) was used. Injection sample concentration was $0.25 \mathrm{mg} / \mathrm{mL}$ in total. The flow rate of mobile phase was $0.3 \mathrm{~mL} / \mathrm{min}$. 


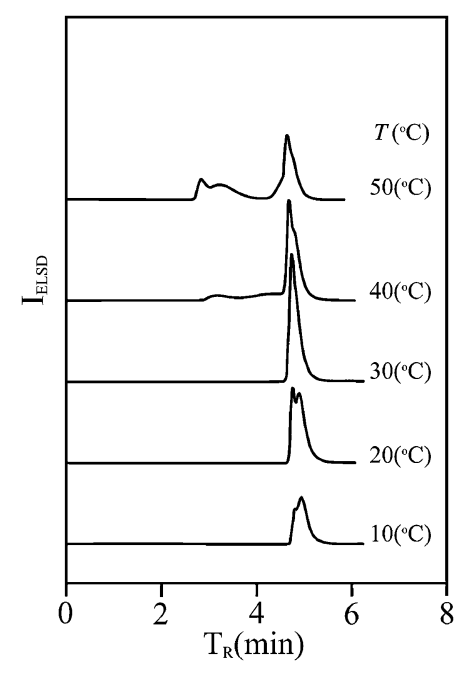

Figure 2. Temperature effect on the retention of 4 PMMA standard samples by system I. The chromatograms obtained by the isocratic mixture $(1,4$-dioxane $/ n$-hexane $=66 / 34)$ and isothermal elutions at different temperatures as labeled in the figure. Otherwise, separation conditions are the same as in Figure 1.

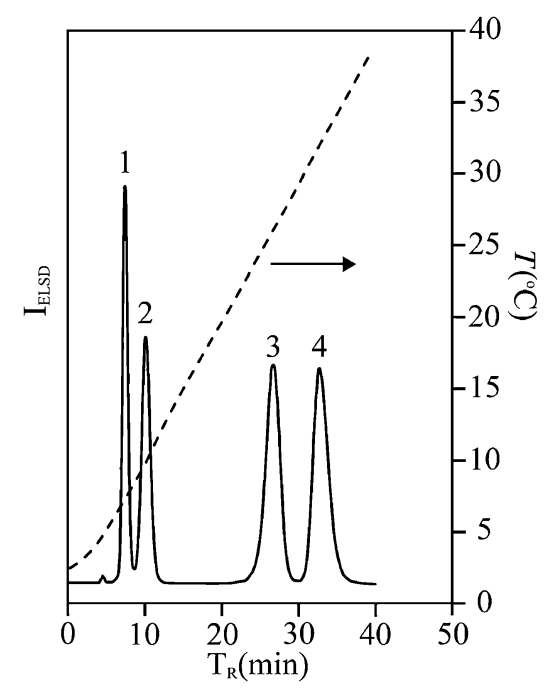

Figure 3. NP-TGIC chromatogram of 4 PMMA standard samples by System I. The chromatogram was obtained by the isocratic elution $(1,4$-dioxane $/ n$-hexane $=66 / 34)$. Temperature gradient was shown in the figure. The sample numbers are listed in the Table I. The sample solution was made at the concentration of about $0.25 \mathrm{mg} / \mathrm{mL}$ for each standard. A cyano-modified silica column (Waters, $\mu$-bondasphere, particle size $5 \mu \mathrm{m}$, pore size $10 \mathrm{~nm}$, $150 \times 3.9 \mathrm{~mm} \mathrm{I.D.)}$ was used. The flow rate of mobile phase was $0.3 \mathrm{~mL} / \mathrm{min}$.

weight fractionation. In Figure 3, TGIC chromatogram obtained by a positive temperature gradient from $2{ }^{\circ} \mathrm{C}$ to $38^{\circ} \mathrm{C}$ (shown by dotted line in the right ordinate) in System I is shown. The eluents for TGIC analysis is the isocratic mixture of near the critical composition (1,4-dioxane $/ n \mathrm{Hx}=66 / 34, \quad \mathrm{v} / \mathrm{v})$ at $30^{\circ} \mathrm{C}$. The sample solution was homogeneous and clear solution even at the lowest temperature. The

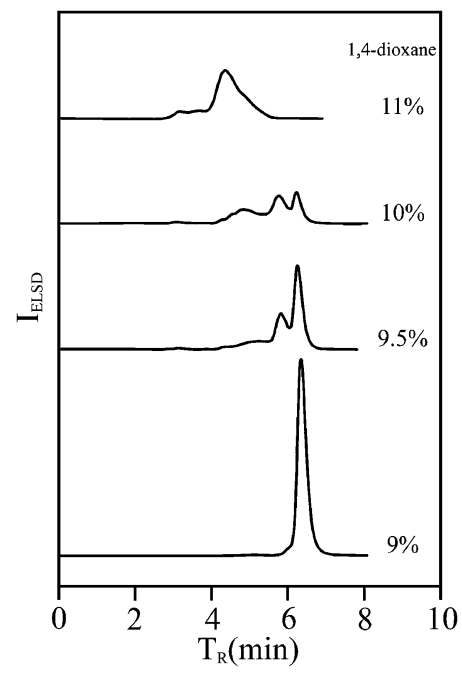

Figure 4. Solvent composition effect on the chromatograms of 4 PMMA standard samples by System II. The isocratic elution of 1,4-dioxane $/ \mathrm{CH}_{2} \mathrm{Cl}_{2}$ at different compositions as labeled in the figure were tested at a constant temperature, $20^{\circ} \mathrm{C}$, to find the critical condition. A bare silica column (Chemco, Nucleosil, particle size $7 \mu \mathrm{m}$, pore size $10 \mathrm{~nm}, 150 \times 4.0 \mathrm{~mm}$ I.D.) was used. Injection sample concentration was $0.25 \mathrm{mg} / \mathrm{mL}$ in total. The flow rate of mobile phase was $0.3 \mathrm{~mL} / \mathrm{min}$.

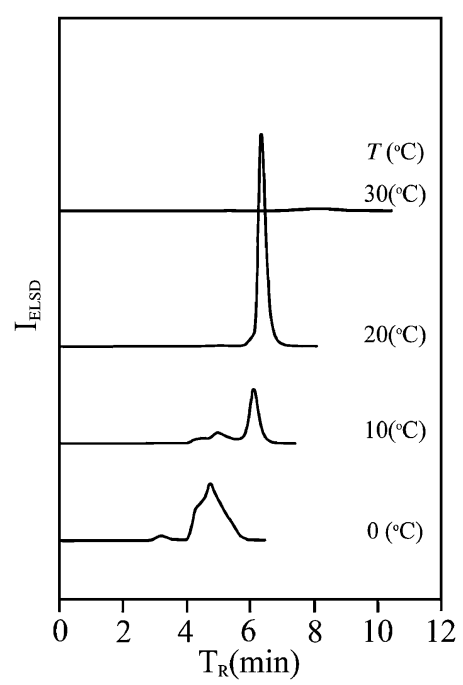

Figure 5. Temperature effect on the retention of 4 PMMA standard samples by System II. The chromatograms obtained by the isocratic mixture (1,4-dioxane $\left./ \mathrm{CH}_{2} \mathrm{Cl}_{2}=9 / 91\right)$ and isothermal elutions at different temperatures as labeled in the figure. Otherwise, separation conditions are the same as in Figure 4.

sample concentration was about $0.25 \mathrm{mg} / \mathrm{mL}$ for each standard. All samples were separated in the order of molecular weight. By using System II, similar experiments were carried out.

In Figures 4 and 5, the solvent effect and temperature effect on System II are shown, respectively. To find the critical composition, the mixture of 1,4-dioxane and $\mathrm{CH}_{2} \mathrm{Cl}_{2}$ at various compositions were tested 


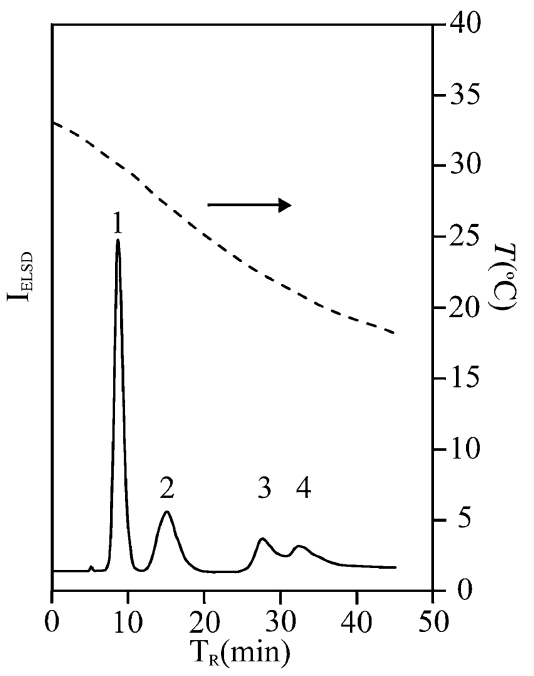

Figure 6. NP-TGIC chromatogram of 4 PMMA standard samples by System II. The chromatogram was obtained by the isocratic elution (1,4-dioxane $/ \mathrm{CH}_{2} \mathrm{Cl}_{2}=9 / 91$ ). Temperature gradient was shown in the figure. The sample numbers are listed in the Table I. The sample solution was made at the concentration of about $0.25 \mathrm{mg} / \mathrm{mL}$ for each standard. A bare silica column (Chemco, Nucleosil, particle size $7 \mu \mathrm{m}$, pore size $10 \mathrm{~nm}, 150 \times 4.0 \mathrm{~mm}$ I.D.) was used. The flow rate of mobile phase was $0.3 \mathrm{~mL} / \mathrm{min}$.

at a constant temperature, $20^{\circ} \mathrm{C}$. It was found that the eluent containing 9 vol. \% of 1,4-dioxane appeared to be around the critical composition. At higher temperature, the samples were retained strongly, whereas at the lower temperature, all samples were eluted in the SEC regime. Therefore, it was found that the negative temperature gradient was effective for TGIC in System II. As clear from the chromatogram shown in Figure 6, all samples were separated with negative temperature gradient from $33^{\circ} \mathrm{C}$ to $18^{\circ} \mathrm{C}$. The peaks of standards 3 and 4 look low compared with those in Figure 3. This may suggest lower recovery due to the strong adsorption in the pores. However, since the positions of the peaks agreed to those by single injections of the respective samples, the low recoveries do not affect the results where the samples were separated by the negative temperature gradient. We could found that both positive and negative temperature gradients were effective in the NP-TGIC for PMMA.

In the theory of interactive polymer chromatography (IPC) using porous packing materials, retention volume $\left(V_{\mathrm{R}}\right)$ of the polymer sample is:

$$
V_{\mathrm{R}}=V_{0}+K V_{\mathrm{P}}
$$

where $V_{0}$ is interstitial volume, $K$ and $V_{\mathrm{P}}$ is partition coefficient and pore volume, respectively. The first theoretical analysis of IPC has been reported twenty years ago by Belenky et al. ${ }^{14}$ In the context of this theory, the partition coefficients $K_{D}$ (pore diameter D) are subdivide into two parts: $:^{15}$

$$
K_{D}=K_{D, S E C} K_{D, I N T}
$$

where $K_{D, S E C}$ is the contribution from the size exclusion effect and $K_{D, I N T}$ is that from the adsorptive interaction. These coefficients are related to the thermodynamical functions:

$$
K_{D, S E C}=\exp \left(\Delta S_{\text {conf }} / k_{\mathrm{B}}\right)
$$

and

$$
K_{D, I N T}=\exp \left(-\Delta E / k_{\mathrm{B}} T\right)
$$

where $\Delta S_{\text {conf }}$ is the difference in polymer conformation entropy (per macromolecule) between confined and unconfined states, $k_{\mathrm{B}}$ and $T$ are Boltzmann constant and absolute temperature. $\Delta E$ represents the local change of free energy of the system (in a theoretical plate) associated with adsorption per a macromolecule, which consists of not only local enthalpy change of adsorption ( $\Delta H_{\mathrm{ad}}$ ) but also local entropy change $\left(\Delta S_{\text {ad }}\right)$ due to the fact that a certain amount of repeating units replace the solvent molecules near the surface and create reversible bonds with the active sites of the adsorbent surface. Under the interactive condition, $\Delta E$ must be negative as follows:

$$
\Delta E=\Delta H_{\mathrm{ad}}-T \Delta S_{\mathrm{ad}}<0
$$

If the resulting effective energy for one such bond (segment interaction energy) is $\varepsilon$, and the equilibrium fraction (per Dalton) of monomer units having temporary bonds with active sites on the surface is $\theta$, then $\Delta E=M \theta \varepsilon$, where $M$ is molecular weight of the macromolecule. Generally, partition coefficient $\left(K_{D}\right)$ is also related to the change of Gibbs free energy $\left(\Delta G_{\mathrm{R}}\right)$ for the retention:

$$
\Delta G_{\mathrm{R}}=-R T \ln K_{D}=\Delta E-T \Delta S_{\mathrm{conf}}
$$

The capacity factor $\left(k^{\prime}\right)$ is related to $K_{D}$ as follows:

$$
k^{\prime}=\frac{V_{\mathrm{R}}-V_{0}}{V_{0}}=\frac{K_{D} V_{\mathrm{P}}}{V_{0}}=K_{D} \phi \quad \phi=V_{\mathrm{P}} / V_{0}
$$

From the eq 6,7 and Gibbs-Helmholtz equation $\{\partial(\Delta G / T) / \partial T\}_{P}=-\Delta H / T^{2}$, the variation of the capacity factor with temperature $\left(\partial \ln k^{\prime} / \partial T\right)_{P}$ can be written as follows:

$$
\begin{aligned}
\left(\frac{\partial \ln k^{\prime}}{\partial T}\right)_{P} & =-\left\{\frac{\partial\left(\Delta G_{\mathrm{R}} / T\right)}{\partial T}\right\}_{P} \cdot \frac{1}{R}+\left(\frac{\partial \ln \phi}{\partial T}\right)_{P} \\
& =\frac{\Delta H_{\mathrm{ad}}}{R T^{2}}+\left(\frac{\partial \ln \phi}{\partial T}\right)_{P}
\end{aligned}
$$

If the terms of $(\partial \ln \phi / \partial T)_{P}$ is negligible, $\left(\partial \ln k^{\prime} / \partial T\right)_{P}$ and also $\left(\partial V_{\mathrm{R}} / \partial T\right)_{P}$ have same sign as $\Delta H_{\mathrm{ad}}$. Therefore, if using the positive temperature gradient, $\left(\partial V_{\mathrm{R}} / \partial T\right)_{P}<0$, the sign of $\Delta H_{\mathrm{ad}}$ is negative, while if using the negative temperature gradient, $\left(\partial V_{\mathrm{R}} /\right.$ $\partial T)_{P}>0$, the sign of $\Delta H_{\mathrm{ad}}$ is positive. 
In many cases of TGIC reported, direction of temperature gradient is positive and $\Delta H_{\mathrm{ad}}$ is negative (exothermic adsorption), and also our present System I is the same, while a few cases of endothermic adsorption $\left(\Delta H_{\mathrm{ad}}>0\right)$ were reported for both RPand NP-mode. In the RP-mode, PEO samples in the aqueous eluents were separated with a negative temperature gradient. ${ }^{2-4}$ In the NP-mode, PS samples in the organic eluents were separated with a negative temperature gradient, as reported in our previous paper. ${ }^{6}$ In the present case, PMMA in System II was also separated with a negative temperature gradient. From eq 5, $\Delta E$ must have negative sign under the interactive condition. If $\Delta H_{\mathrm{ad}}>0$ under this condition, $\Delta H_{\text {ad }}$ has to be smaller than $T \Delta S_{\text {ad }},\left(0<\Delta H_{\text {ad }}<\right.$ $T \Delta S_{\text {ad }}$ ), and then $\Delta S_{\text {ad }}$ is positive. In System II in the present work and also in System I and II in our previous paper for PS, ${ }^{6}$ the separation of samples were carried out by the negative temperature gradient. In these cases, $\Delta H_{\text {ad }}$ and $\Delta S_{\text {ad }}$ must be positive and $0<$ $\Delta H_{\text {ad }}<T \Delta S_{\text {ad }}$ from eq 5, though these were not observed directly. The similar result was observed in the RP-mode separation for PEO in the aqueous eluents by Cho et al. ${ }^{4}$ They obtained $\Delta H_{\text {ad }}>0$ and $\Delta S^{*}>0$ $\left(\Delta S^{*}=\Delta S^{\circ} / R+\ln \phi, \Delta S^{\circ}=\Delta S_{\text {ad }}+\Delta S_{\text {conf }}\right.$ in our representation) from van't Hoff plot $\left(\ln k^{\prime} \sim 1 / T\right)$ and explained this phenomenon by the effect of hydrophobic interaction. In these cases of positive $\Delta H_{\mathrm{ad}}$, the adsorption of the macromolecules is not enthalpy-driven process but entropy-driven process.

On the difference between our Systems I and II, our speculation from molecular viewpoint is as follows: Both adsorbents have high polar active site on the surface and the eluent promoting polymer desorption is the same 1,4-dioxane. On the other hand, the eluents promoting polymer adsorption are different between both systems. In system II, the repeating units of PMMA replace $\mathrm{CH}_{2} \mathrm{Cl}_{2}$ molecules having reversible bonds with the active sites on the surface and create new reversible bonds instead. Positive $\Delta H_{\mathrm{ad}}$ is understandable taking into consideration that the endothermic contribution to $\Delta H_{\mathrm{ad}}$ by displacing $\mathrm{CH}_{2} \mathrm{Cl}_{2}$ molecules may be larger than the exothermic contribution due to adsorption of the repeating units. The entropy increase caused by displacing $\mathrm{CH}_{2} \mathrm{Cl}_{2}$ molecules from the surface is larger than the entropy loss due to adsorption of the repeating units, since the units are interlinked in polymer molecules and then ordered at some degree even before adsorption. Then, $\Delta S_{\mathrm{ad}}$ is positive. On the other hand, in System I, $\Delta H_{\text {ad }}$ is negative because the replacement of polar groups of the repeating units with $n \mathrm{Hx}$ molecules on the polar surface is exothermic process. The entropy increase is not expected, even if the repeating units of PMMA replace $n \mathrm{Hx}$ molecules in the vicinity of the surface, since the molecules of $n \mathrm{Hx}$ have not specific interaction with the active site differently from $\mathrm{CH}_{2} \mathrm{Cl}_{2}$ molecules.

According to the molecular theory by Gorbunov and Skvortsov, ${ }^{16,17}$ the distribution coefficients $K$ of flexible chain macromolecules are, in general, described by two dimensionless parameters: the ratio $(g)$ of the sizes of the macromolecule $\left(R_{\mathrm{g}}\right)$ and the pore (d), $g=R_{\mathrm{g}} / d$ and the ratio $(\lambda)$ of the pore size to the correlation length of adsorption $(L), \lambda=-d / L$. The physical meaning of $L$ (denoted by $\mathrm{H}$ in the original paper) is the mean thickness of the adsorption layer near surface in the adsorbent pores, and is positive under the condition of adsorption. They deduced the relationship between $L$ and $\varepsilon$ or temperature near the critical condition from the assumption of de Gennes': ${ }^{18}$

$$
L \sim \frac{b}{\varepsilon_{\mathrm{cr}}-\varepsilon} \sim \frac{b}{T-T_{\mathrm{cr}}}
$$

where $b$ is the size of the unit (segment) of the chain, and $\varepsilon_{\mathrm{cr}}$ and $T_{\mathrm{cr}}$ are the critical energy and the critical temperature, respectively. The critical conditions are defined as the conditions in which the entropic losses of the chain in the pore are exactly compensated by the energy gain from adsorption of the units on the pore walls $\left(\Delta E=T \Delta S_{\text {conf }}\right)$. Under the condition of adsorption, $-\varepsilon>-\varepsilon_{\mathrm{cr}}$. Eq 9 represents only the case of the negative temperature gradient. If the temperature gradient is positive, eq $9^{\prime}$ should be used instead of eq 9 .

$$
L \sim \frac{b}{\varepsilon_{\mathrm{cr}}-\varepsilon} \sim \frac{b}{T_{\mathrm{cr}}-T}
$$

For the above mentioned cases of positive temperature gradients, this equation is valid.

\section{CONCLUSION}

In NP-mode of TGIC, it was found that both cases of positive and negative temperature gradients were effective for PMMA samples. That is, as well as PS samples in our previous paper, ${ }^{6}$ PMMA samples were separated by the negative temperature gradient using a bare silica column and the mobile phase of 1,4-dioxane and $\mathrm{CH}_{2} \mathrm{Cl}_{2}$ (System II), and also by the positive temperature gradient using a cyano-modified silica column and mobile phase of 1,4-dioxane and $n \mathrm{Hx}$ (System I).

\section{REFERENCES}

1. H. C. Lee and T. Chang, Polymer, 37, 5747 (1996).

2. C. H. Lochmüller, M. A. Moebus, Q. Liu, C. Jiang, and M. Elomaa, J. Chromatogr. Sci., 34, 69 (1996). 
3. T. Andersen, P. Molander, R. Trones, D. R. Hegna, and T. Greibrokk, J. Chromatogr., A, 918, 221 (2001).

4. D. Cho, S. Park, J. Hong, and T. Chang, J. Chromatogr., A, 986, 191 (2003).

5. W. Lee, D. Cho, B. O. Chun, T. Chang, and M. Ree, J. Chromatogr., A, 910, 51 (2001).

6. S. Teramachi, H. Matsumoto, and T. Kawai, J. Chromatogr., A, 1100, 40 (2005).

7. S. Park, C. Ko, H. Choi, K. Kwon, and T. Chang, J. Chromatogr., A, 1123, 22 (2006).

8. M. B. Tennikov, P. P. Nefedov, M. A. Lazareva, and S. Y. Frenkel', Vysokomol. Soyed. A, 19, 657 (1977).

9. S. Mori and Y. Uno, J. Appl. Polym. Sci., 34, 2689 (1987).

10. A. A. Gorbunov, L. Y. Solovyova, and A. M. Skvortsov, Polymer, 39, 697 (1998).
11. M. Janco, D. Berek, A. Önen, C.-H. Fischer, Y. Yagci, and W. Schnabel, Polym. Bull., 38, 681 (1997).

12. D. Berek, M. Janco, and G. R. Meira, J. Polym. Sci., Part A: Polym. Chem., 36, 1363 (1998).

13. D. Berek, M. Janco, K. Hatada, T. Kitayama, and N. Fujimoto, Polym. J., 29, 1029 (1997).

14. B. G. Belenky, E. S. Gankina, M. B. Tennikov, and L. Z. Vilenchik, J. Chromatogr., 147, 99 (1978).

15. Y. Brun, J. Liq. Chromatogr. Relat. Technol., 22, 3027 (1999).

16. A. A. Gorbunov and A. M. Skvortsov, Vysokomol. Soyed. A, 28, 2170 (1986).

17. A. M. Skvortsov and A. A. Gorbunov, Vysokomol. Soyed. A, 31, 1194 (1989).

18. P.-G. de Gennes, Rep. Prog. Phys., 32, 187 (1969). 